Voix et Images

voixetimages

\title{
Entretien avec Michel Marc Bouchard
}

\section{Shawn Huffman}

Volume 33, numéro 1 (97), automne 2007

Michel Marc Bouchard

URI : https://id.erudit.org/iderudit/017524ar

DOI : https://doi.org/10.7202/017524ar

Aller au sommaire du numéro

Éditeur(s)

Université du Québec à Montréal

ISSN

0318-9201 (imprimé)

1705-933X (numérique)

Découvrir la revue

Citer ce document

Huffman, S. (2007). Entretien avec Michel Marc Bouchard. Voix et Images, 33(1),

15-25. https://doi.org/10.7202/017524ar d'utilisation que vous pouvez consulter en ligne.

https://apropos.erudit.org/fr/usagers/politique-dutilisation/ 


\author{
$+++$ \\ SHAWN HUFFMAN \\ Université du Québec à Montréal
}

voIX ET IMAGES On fait remarquer souvent le très jeune âge auquel vous avez commencé à faire du théâtre: une première pièce mise en scène à l'âge de 14 ans, votre implication dans la compagnie de thêâtre de votre cégep à Matane, le coup de cœur définitif pour les planches au sortir de vos études collégiales en tourisme. Pourrait-on remonter encore plus loin, à votre enfance même, pour chercher l'origine de cet engouement?

Michel MARC BOUCHARD On a une façon différente de voir, chaque décennie, les origines mêmes des raisons pour lesquelles on écrit. Mais pour moi, il y a toujours eu le besoin de parler à quelqu'un qui n'est pas là. Ce besoin vient en grande partie de la mort de ma sœur. Elle avait trois ans et demi, j'en avais six et je me souviens qu'après son décès je lui parlais beaucoup. Tout s'est compliqué quand j'ai commencé à croire qu'elle me répondait...

voIX ET IMAGES Comme une sorte de fantôme ou bien comme une absence?

MICHEL MARC BOUCHARD Plutôt une absence. Pour quelqu'un qui est profondément catholique, il y a cette croyance d'une sorte de substrat après la mort. C'est comme si elle était présente et à un moment donné j'ai eu des conversations avec elle. Et puis, je viens de l'oralité, je ne viens pas de la littérature. Comme je l'ai raconté mille fois, je viens d'une famille très nombreuse. Ma grand-mère s'est mariée quatre fois, ce qui fait qu'il y avait énormément de gens qui vivaient dans la maison de mon grand-père. Les conteurs, et plus particulièrement les conteuses, y faisaient l'animation. Chez nous, quelqu'un qui ne savait pas raconter était quelqu'un de mort. C'était vraiment une tradition d'oralité; on racontait tout: les souvenirs d'antan, le quotidien... Ceux qui avaient la meilleure répartie, la parole facile, étaient le centre d'attraction. Disons que j'ai été à bonne école.

voIX ET IMAGES C'était une mise en scène de la parole...

MICHEL MARC BOUCHARD Absolument. Face à un groupe déjà nombreux! Le dimanche, on pouvait facilement se retrouver à trente, quarante, cinquante personnes. Car tout le monde venait visiter mon grand-père et ma grand-mère. Ce goût pour le théâtre s'est manifesté dès l'école primaire. Des professeurs m’ont rappelé que je faisais déjà partie de tous les spectacles et que j'avais même la prétention de vouloir les organiser. Avec des cousins et des cousines, je faisais des séances aussi, ce que nous appelions des séances. C'était moi qui faisais la distribution. Ce que mes cousins 
comprenaient mal, c'était qu'on répétait le lendemain ce qu'on avait présenté la veille. C'est dire que déjà très tôt dans ma vie cette dimension ludique était présente. VoIX ET IMAGES Vous êtes né à la fin des années cinquante, une période de profondes transformations au Québec, une période de laïcisation radicale. Pourtant, l'Église et ses images, ses symboles, traversent votre thêâtre. Pourriez-vous décrire le début de votre parcours theâtral à la lumière de ce premier théâtre, c'est-à-dire l'Église et ses cérémonies, voire son théâtre?

MICHEL MARC BOUCHARD Les mythes auxquels j'ai été confronté sont ceux entourant la religion, transmis et représentés par la parole du rituel de la messe. Il y avait là quelque chose de complètement irréel et de profondément exotique, surtout pour quelqu'un du Lac-Saint-Jean. On nous racontait ce qui s'était passé en Judée, à Jérusalem. Les noms des personnages de ces histoires ne ressemblaient à aucun des noms de chez nous! C'était donc mon premier rapport à la littérature et aux histoires fantastiques. C'est toute cette mythologie catholique qui, d'une certaine façon, m'a interpellé. Car cette époque des années cinquante, de la Révolution tranquille, concerne une élite qui faisait bouger les choses, mais dans les grands centres. Ma mère et mon père, étant les derniers de grandes familles - chez ma mère, ils étaient douze et chez mon père, neuf - , ont vécu différemment ce passage. Ils ont pu exprimer leur révolte par rapport à ces changements et en même temps la raconter. Et moi, j'ai hérité d'un peu tout ça. À mon âge, j'ai vu la Fête-Dieu, j'ai vu ce que c'était, Pâques, avec tout son cérémonial. Et ma mère en particulier a parlé de cette époque, de la Grande Noirceur, où l'Église déterminait tout. Les années cinquante, c'est une époque très importante, le passage d'une ère à une autre, au Québec. Il ne faut pas oublier que j'ai fait le séminaire. J'ai étudié quatre ans au Séminaire de Métabetchouan. C'était un séminaire construit à la fin des années 1960 et qui était plus moderne, une très belle école. J'étais donc éduqué en grande partie par les prêtres qui véhiculaient toutes ces valeurs. Ma mère, cependant, me disait souvent: "S'ils te demandent si tu veux être prêtre, réponds "je ne sais pas", comme ça tu finiras tes études!» Au séminaire aussi, j'ai fait du théâtre.

voIX ET IMAGES Est-ce que le personnage du père Saint-Michel dans Les feluettes provient justement de cette expérience du séminaire?

MICHEL MARC BOUCHARD Ce qui me fascine aujourd'hui encore de la vie au séminaire, c'est la codification qu'il y avait de toutes ces choses et, surtout, de l'abstrait.

VoIX ET IMAGES Il y a quand même une transformation, une transposition que vous faites dans votre thêâtre. Vous ne prenez pas ces symboles tels quels. Vous les modifiez; vous leur accordez un autre sens.

MICHEL MARC BOUCHARD Je pense que les pièces qui sont les plus claires par rapport à ça, c'est Les feluettes et Le peintre des madones, où je récupère les symboles religieux de manière extrêmement érotique. Le symbole de Saint Sébastien est lié à la sensualité sadomasochiste. Il a été aussi une icône du mouvement gai, du martyre, entre autres, mais aussi du sacrifice amoureux. Dans Le peintre des madones, l'image de la Vierge est complètement érotisée et le personnage du prêtre vit une sorte de voyage sacrificiel dans la souffrance de la chair. Par ailleurs, il y a aussi tout le cérémonial dans la religion. Quand on va à l'église, il y a la cérémonie, oui, mais il y a aussi une scène, une scène à l'italienne, sur laquelle il y aura de la parole et de 
l'action. C'est très fascinant de faire dire à cet homme en pagne sur la croix, les bras ouverts et qui saigne: " voici mon corps, livré pour vous, mangez-le; voici mon sang, il est pour vous, buvez-le», dans une société très pudique, où le corps, la nudité n'existent pas.

voIX ET IMAGES Je pensais aussi à L'histoire de l'oie et aux Yeux de verre que j'ai vues récemment et dans lesquelles on retrouve ces objets ecclésiastiques: la maisonchâsse dans la première et le présentoir contenant la poupée dans la deuxième, ce présentoir qui rappelle le cercueil de verre servant à exposer et à préserver les saints dans l'église.

MICHEL MARC BOUCHARD Ce sont des choix scénographiques qui ne figurent pas dans les didascalies. Dans Des yeux de verre, tout ce que je dis c'est que les poupées sont dans une armoire vitrée. Ce que je propose comme scénographie est beaucoup plus de l'ordre de la morgue: une série de petites boîtes, sur lesquelles il y a des numéros, des noms de poupées, des noms de membres et tout. Comme une espèce de grand charnier...

voIX ET IMAGES Tout comme Jeanne-Mance Delisle pour l'Abitibi ou encore Daniel Danis pour le Saguenay, votre thêâtre évoque les paysages, les mœurs et le langage de votre région natale, le Lac-Saint-Jean.

Michel MARC Bouchard Je pense que l'on crée d'où l'on vient. Le Lac-Saint-Jean est une région assez fermée, assez isolée, assez chauvine, mais qui a sa propre mythologie, ses propres histoires, ses héros, ses personnages. C'est un monde en soi, à cause de l'isolement, un monde extrêmement riche auquel je suis encore très attaché. Prenons simplement comme exemple l'Hôtel Roberval, qui sert de cadre aux Feluettes: les aristocrates qui sont débarqués là, complètement paumés, côtoient de riches Américains, des touristes de partout dans le monde, des Amérindiens et la population locale. Il y avait assez de matière pour écrire une épopée! Aussitôt que l'on cherche, on trouve dans chaque village assez de matière pour une représentation plus symbolique, plus mythique même. Toutefois, en ce qui concerne la langue, je n'ai jamais été capable de reproduire le jeannois. D'abord parce que, en tant qu'écrivain, je trouve que son vocabulaire est assez pauvre et puis, parce que c'est une langue qui, contrairement à l'acadien qui a des sonorités très typiques, se distingue plutôt par ses tonalités. Ce n'est pas une syntaxe particulière, mais plutôt une façon qu'ont les gens de chanter la langue, une ligne mélodique. Et puis on ajoute «là là» à chaque mot, alors s'il fallait que je reproduise cette langue mes textes seraient deux fois plus longs! Par contre, je conserve certains traits du vocabulaire, comme cette façon de dire "papa» ou "maman» ou encore certains mots. Mais de façon générale, j'aurais trouvé ça un peu maniéré d'employer uniquement cette langue. J'écris plutôt dans un français assez standard, même si dans Des yeux de verre, je suis revenu plus près d'un français québécois. Mais dans Le peintre des madones, par exemple, il n'y aurait aucune transposition à faire pour une scène française.

VoIX ET IMAGES Parce que vous employez ce mot, "transposition », parlons justement de cette fameuse «traduction» en français hexagonal des Muses orphelines.

MICHEL MARC BoUCHARD Pour moi, cette traduction constitue un pari politique plutôt qu'esthétique. À l'époque se tenait au Québec un débat sur les raisons pour 
lesquelles le théâtre québécois n'était pas joué à Paris. Le principal obstacle était la langue. Des productions françaises avaient même essayé de reproduire l'accent québécois. C'était lamentable, maniéré et absurde. Alors à l'initiative du CEAD [Centre des auteurs dramatiques] est né ce projet de proposer non pas une adaptation, non pas de simples aménagements, mais bien une véritable traduction. J'ai donc travaillé avec Noëlle Renaude, une auteure reconnue, une auteure à part entière qui a donc pris une liberté d'auteure. Mais une traduction à l'intérieur d'une même langue fait face aux nombreux écueils, que créent, entre autres, les faux amis. Une traduction en langue étrangère doit respecter non seulement le sens et l'efficacité dramatique, mais surtout le rythme. Le traducteur doit traduire mon souffle. Or, on ne retrouve pas ce souffle-là dans la version de Noëlle. Un seul exemple serait celui de la négation, où elle conserve le «ne» alors qu'ici nous en faisons l'ellipse. Et il faut en même temps trouver des référents français qui ne sont pas vraiment d'ici. Enfin, il est fabuleux de voir que la production du Théâtre le Dilettante, en France, il y a trois ans, a eu un tel succès parce qu'ils ont décidé de jouer la pièce originale, sans parti pris d'accent.

voIX ET IMAGES Ce qui m'a fait réagir dans la version française des Muses orphelines, c'était justement le mot «traduction», qui donne une impression de mépris envers le français parlé au Québec.

Michel MARC BouchaRd Pour l'anecdote... à Nantes, dans un théâtre que je ne nommerai pas, des acteurs étaient en train de répéter Les muses orphelines. Noëlle est allée les voir et m'a appelé ensuite pour me dire à quel point elle était outrée. Sauf pour le personnage d'Isabelle, ils avaient pris la version française de la pièce; Isabelle, elle, parlait en québécois. Pourquoi? Parce qu'on la surnomme «La débile». Pour moi, ça a été une véritable gifle. Mais il faut admettre que ça a beaucoup bougé depuis. Il y a une forte présence québécoise en France maintenant et notre théâtre commence à être lu, à être entendu, à être joué dans son écriture originale.

VoIX ET IMAGES Même si votre théâtre pousse dans une terre riche et fertile, décrite d'ailleurs dans Les feluettes ou encore dans Les porteurs d'eau, toute tentative de rapprochement avec le terroir, c'est-à-dire cette célébration naïve de la terre et de l'habitant, y est vouée à l'échec, car les rapports interpersonnels, surtout ceux du cercle familial, sont presque toujours hostiles, violents même. Cette violence se trouve un peu partout dans votre œuvre: de La contre-nature de Chrysippe Tanguay, écologiste à votre pièce la plus récente, Des yeux de verre.

MICHEL MARC BOUCHARD Le thêâtre est conflit. Quel que soit le milieu, il suppose le plus souvent une approche conflictuelle. La région comme telle n'est pas toujours conflictuelle dans mes pièces. Elle ne l'est pas, par exemple, dans Les feluettes où ce sont les mentalités et l'époque qui sont conflictuelles. Au contraire, la région y est presque magnifiée dans ce qu'elle a à offrir comme environnement naturel. Dans Les muses orphelines, on parle beaucoup d'isolement. Ce n'est pas une attaque, là non plus, contre la région; on parle plutôt de son isolement ou de son inculture, voire de sa xénophobie. Là où j'ai peut-être été le plus dur, c'est dans Le chemin des Passesdangereuses. Ambroise parle de son angoisse quand il lui faut y revenir. La pièce montre cette tension Montréal-Québec-Région que j'ai même un peu exagérée, car j'en avais assez d'entendre parler DU Québécois alors qu'il y en a plein de types de 
Québécois; chacun a sa planète, son langage, sa culture. Dans la pièce, on voit la confrontation entre trois types d'hommes québécois qui sont pourtant originaires d'un seul et même endroit, d'une même famille. Aujourd'hui, les familles sont dispersées. Il est très rare de voir des familles qui sont toujours réunies dans un même territoire. Le conflit entre Karl et Ambroise repose sur les différences de perception que chacun a de la région. Karl en a une vision très positive, alors qu'Ambroise en a une vision très dure qui découle de son enfance; il a été victime d'ostracisme d'abord parce qu'il était gai, puis parce qu'il appartenait au milieu artistique.

voix ET IMAGes Dans ses Journées de lecture, Proust évoque avec nostalgie la découverte de la lecture par l'enfant. Les enfants et les personnages enfantins dans votre œuvre ne sont pas nostalgiques. Au contraire, votre théâtre représente souvent un adulte aux prises avec ses souvenirs d'enfant. Dans sa pièce Tu faisais comme un appel, Marthe Mercure a déjà employé le théâtre à des fins thérapeutiques pour amener des orphelines de Duplessis à briser le silence sur leur passé. Quel est le rôle de l'enfant dans votre théâtre? Et est-ce que ce théâtre, de manière plus intime peut-être et certainement de façon moins directe, mobilise des stratégies thérapeutiques lui aussi?

MICHEL MARC BOUCHARD La dimension thérapeutique de mon théâtre reste non avouée même s'il est aussi nourri de préoccupations qui sont de l'ordre du vécu. Le vécu, en passant, c'est ce qu'on a subi, ce qu'on a vu, ce qui nous a révolté, ce qu'on a observé et même, des fois, avec l'intention de changer des choses. Il y a deux ans, quand j'ai commencé à retravailler Des yeux de verre, je me suis aperçu avec étonnement, peut-être même candidement - ce qui n'est pas du tout négatif - , qu'il y avait là le germe de plusieurs de mes autres œuvres, notamment du désir de Chrysippe de porter un enfant, des Feluettes où l'amour adolescent est réprimé, de l'enfance abandonnée des Muses orphelines à l'enfance trahie du Voyage du couronnement, à l'enfance violentée de L'histoire de l'oie. Cette récurrence de la violence envers l'enfant vient certainement d'une blessure, car il y a une blessure en moi. Mais je n'en parlerai jamais en public. Toujours est-il que tout cela, quand j'écris, reste de l'ordre de l'inconscient. Ce n'est donc pas un thêâtre thérapeutique dans le sens propre du terme - sauf peut-être pour L'histoire de l'oie -, mais plutôt un théâtre qui conserve les traces d'une blessure.

voIX ET IMAGES En lisant vos pièces, on se rend compte que la culture populaire télévisuelle et le cinéma alimentent votre imaginaire, surtout dans vos pièces sur l'amour gai: je pense à la référence à la "comtesse à la triste robe» [Angélique, marquise des Anges] dans Les feluettes ou encore à cette référence à Leonard Chow et à Damon D'Oliveira, deux acteurs de télévision, au début du Jade et l'ébène.

MICHEL MARC BOUCHARD Je viens d'une culture populaire. J'étais peut-être un peu snob pour les gens de mon village, mais je viens quand même de cette culture. Contrairement à La contre-nature, qui est une pièce extrêmement conceptuelle, le besoin d'écrire Les feluettes est venu du désir de raconter une histoire d'amour que l'on ne m'avait jamais racontée parce que toutes les histoires d'amour emploient, pour nous, les gais, un vocabulaire emprunté. Puis est arrivée l'étape des prisonniers qui jouaient tous les rôles. J'ai déjà vu des productions où les rôles sont joués par des femmes. Ça ne fonctionnait pas du tout. C'était kitsch. Le fait que ce soit juste 
des hommes crée une sorte de transfert qui, si c'est bien joué, est loin de la caricature. Et ce, malgré le fait que pour écrire cette histoire romantique, j'ai dû chercher des références un peu kitsch. Un peu soap, un peu grandiloquentes. Parce que le romantisme est un produit d'exportation, mis à part peut-être certains auteurs du XIXe siècle québécois qui ont tenté d'imiter des auteurs français. C'est la raison pour laquelle j'ai décidé d'avoir une approche moins convenue. Il fallait aussi rapprocher le public de cette histoire-là. Quand la pièce a été créée, il n'y avait pas autant d'œuvres homosexuelles présentées au public; elle a été produite un an après Being at Home with Claude, et avant ça il n'y avait guère eu qu'Hosanna. Il n'y avait pas eu beaucoup de pièces qui prenaient ce parti pris d'émouvoir. Je voulais aller dans des codes qui n'étaient pas ceux de l'univers du gay underground. Je ne voulais pas dire qu'on était «normal» ou comme tout le monde, qu'on était comme les straights. Je voulais dire que nous éprouvions les mêmes choses. Brassard m'avait dit: "Tu penses que ça va être populaire, mais je pense que ça va se résumer au festival de perfecto et de moustaches. » Mais j'étais convaincu et finalement ça a marché, car autant les homosexuels que les hétérosexuels se sont reconnus dans cette histoire d'amour interdit. C'est le thème de l'amour interdit, je crois, qui a touché une corde sensible chez le public. Ce n'était pas un Roméo et Juliette gai, et de toute façon je crois qu'on a dépassé ce genre d'adaptation-là. Toujours est-il que le contexte y est pour beaucoup. Quand les Japonais ont monté Les feluettes à Tokyo, il y a environ deux ans, ils ont sorti une bande vidéo intitulée Vallier et Simon, pour calquer le titre de Roméo et Juliette. Tout le monde s'est reconnu dans cette histoire d'amour.

voix ET IMAGES Et ces références à Gabriele D'Annunzio qui, dans votre pièce, servent à dire l'amour?

Michel MARC BOUCHARD Ce qui est fascinant, c'est de savoir que Gabriele D’Annunzio a écrit le rôle de Saint Sébastien pour une femme. Mais pour ce qui est de cette différence entre la culture populaire et la culture littéraire ou la Culture avec un grand C, c'est l'accessibilité. Elle n'était pas accessible aux masses, au peuple. Et Simon représente ça. C'est-à-dire qu'il trouve un moyen de comprendre, d'exprimer cet état-là, à travers les mots de cet auteur qui sont presque comme un langage secret, un langage dont eux seuls connaissent la codification. Mais ils savent que c'est leur langage d'amour, même si ça prend neuf scènes pour que Simon avoue qu'il aime Vallier. Il n'était pas capable de le dire, mais à travers D'Annunzio, il peut le dire.

Voix ET IMAGES Vers le milieu des années quatre-vingt, vous avez publié La contrenature de Chrysippe Tanguay, écologiste. Dans cette pièce et dans plusieurs qui suivent, vous soulevez des questions qui touchent non seulement aux personnes gaies, mais à la société en général : l'homoparentalité, le sida, la diversité culturelle, entre autres. Pourquoi, à votre avis, le théâtre québécois, surtout celui des années quatre-vingt, a-t-il été un milieu propice pour débattre de ces questions? Je pense à votre thêâtre, mais aussi à celui de René-Daniel Dubois, d'Alain Fournier, de Jovette Marchessault et de Jeanne-Mance Delisle, entre autres.

MICHEL MARC BOUCHARD Oui et dans bien des cas, j'ai soulevé ces questions avant qu'elles ne deviennent des sujets de débats publics. La contre-nature, c'est en 1984 qu'elle traite de l'homoparentalité. Quelqu'un m'a dit un jour: «Tu te rends compte 
du courage que tu as eu!» Et j'ai dit: «Non, ce n'est même pas une question de courage, c'est une question d'intégrité.» Il faut être intègre pour écrire. Cela explique, en partie, la popularité du thêâtre gai. Les hétérosexuels étaient dans une espèce de malentendu par rapport à la relation de couple, alors que nous, nous n'y étions pas. On s'est permis de parler du désir, on s'est permis de parler de l'amour blessé, de l'amour en devenir aussi. Alors si le théâtre gai de cette époque-là a été si populaire, c'est parce qu'il parlait justement de sujets qui n'étaient pas exclusifs à l'amour gai. On s'est permis de mourir, de souffrir, de vivre, de toucher à l'extase amoureuse, alors qu'eux ne se permettaient plus de mettre en scène le couple, l'amour et la passion.

voIX ET IMAGES Est-ce que vous voyez votre thêâtre aussi comme une tentative de communiquer avec des gais, jeunes ou moins jeunes?

MICHEL MARC BOUCHARD Non, pas vraiment. Il n'y a jamais eu de volonté politique dans mon théâtre. J'ai refusé, par exemple, de faire partie d'une anthologie publiée aux États-Unis sur le théâtre gai. Je refuse l'étiquette. Si mon théâtre devait se résumer à une étiquette, je renoncerais à écrire. Je sais qu'il y a plusieurs auteures qui ont sacrifié leur vie au nom de leurs convictions féministes. Pour moi, il n'en est pas question. Je ne suis pas un auteur nationaliste, je ne suis pas un auteur gai, je suis un auteur. L'étiquette pour moi, c'est un grand piège.

VoIX ET IMAGES Trois de vos pièces ont fait l'objet d'une adaptation: au grand écran dans les cas des Feluettes (John Greyson) et des Muses orphelines (Robert Favreau) et au petit écran dans le cas de L'histoire de l'oie (Tim Southam). Quelle réaction un dramaturge peut-il avoir en voyant ses pièces transposées à l'écran? Ces adaptations cinématographiques ont-elles modifié votre écriture dramaturgique?

MICHEL MARC BOUCHARD Je vois ces adaptations comme des œuvres à part entière. Prenons L'histoire de l'oie, par exemple. Même si le film ne peut avoir la force de la représentation thêâtrale, j'étais quand même satisfait de ce qu'on a fait. C'était facile pour cette pièce-là, car il s'agit d'une narration. Aussi, ce que j'ai trouvé extrêmement touchant, c'était d'avoir un véritable enfant et une véritable oie. Cela fait en sorte que le crime est plus dur, je pense. Ce que j'ai aimé dans l'adaptation des Feluettes, adaptation à laquelle j'ai participé d'ailleurs parce que j'en étais le scénariste, c'était le parti pris du cinéma dans le cinéma dans le cinéma, qui reproduisait le théâtre dans le théâtre dans le théâtre, et aussi le fait que le film est d'une grande thêâtralité. Le film n'a connu que deux problèmes. D’abord celui du budget, parce que les coûts de production étaient évalués à environ sept millions de dollars et on n'en avait que deux. Beaucoup de plans de tournage, de scènes du premier scénario ont disparu, et le personnage de la Comtesse a été énormément réduit parce qu'il fallait se focaliser sur les trois protagonistes. La deuxième difficulté a été d'arrimer ma vision romantique de la pièce à la vision politique de John. La fin du film est très différente de celle de la pièce qui n'est que la suite d'une mise en abyme, parce que John tenait à ne pas laisser comme message aux jeunes gais que si ça ne marchait pas, il fallait se tuer. Au bout du compte, cependant, le film et la pièce restent deux œuvres différentes. C'est aussi le cas des Muses orphelines, film au scénario duquel je n'ai pas participé parce que je trouvais un peu excessif de toujours scénariser mes propres œuvres. Des choix, certains contestables, d'autres adéquats, ont été faits. Le plus significatif était de briser le huis clos, de changer l'époque et de faire disparaître 
toutes les références religieuses. Les trois projets d'adaptation de mes pièces ont été très bien reçus, couverts de prix, même si Les feluettes est celui qui a connu la plus grande carrière. Les gens sont encore fascinés par ce film atypique. On est présentement en négociation pour une adaptation d'une de mes comédies, à savoir Les grandes chaleurs. La scénarisation de la comédie est un exercice que je trouve plus laborieux que les projets précédents, car la pièce est de facture plus classique et repose sur une foule de conventions scéniques.

voIX ET IMAGES Est-ce que le cinéma amène aussi, à l'occasion, un ton naturaliste qui est absent dans votre théâtre? Je pense notamment à l'adaptation de L'histoire de l'oie.

MICHEL MARC BOUCHARD Je crois qu'une simple captation de la représentation n'aurait pas suffi. En particulier à cause de la marionnette et aussi à cause de la distribution. L'acteur qui jouait le petit Maurice était quand même dans la quarantaine! La caméra, c'est un œil qui s'approche au plus près de la scène, qui est plongé dans la scène. C'est pourquoi j'étais d'accord avec les choix de la version filmique. De plus, le réalisateur est un artiste à part entière qui a eu envie d'interpréter luimême l'œuvre. Il n'en est pas l'archiviste. On ne peut pas toujours échapper au naturalisme, à moins qu'il n'y ait une donnée structurelle, comme dans Les feluettes, qui impose une vision plus théâtrale ou plus onirique.

voIX ET IMAGES Est-ce qu'il y a un genre cinématographique qui alimente votre réflexion? Je pense à Koltès, par exemple, à son engouement pour les films de kungfu...

MICHEL MARC BOUCHARD Oui, il y en a plusieurs en fait: les films japonais des années soixante-dix, dont deux de Kurosawa. Il y a aussi Fellini, Visconti, Passolini, Jodorovski, Fassbinder, Arrabal... Ils m'ont tous marqué. Un autre cinéma qui m’a beaucoup parlé est celui de Jutra et de Carle. Les adaptations filmiques de pièces aussi : tout ce qu'on a fait à partir des pièces de Tennessee Williams, par exemple.

voIX ET IMAGES Vous parlez de Fellini... Plusieurs de vos pièces sont traduites en italien justement. Comment expliquez-vous cet engouement pour le théâtre québécois en Italie?

MICHEL MARC BOUCHARD Il est vrai que je suis très joué en Italie; publié aussi. Mais je ne pense pas que je représente une nation, mon théâtre plaît, c'est tout. Il y a des sensibilités qui se reconnaissent dans mon thêâtre et il y a des gens qui ont envie de monter ce que j'ai écrit. Ce qui est fascinant par contre, c'est qu'on peut trouver des pièces qui fonctionnent plus dans certains pays que d'autres. Les plus prisées sont L'histoire de l'oie, Le chemin des Passes-dangereuses et Les muses orphelines. Les feluettes, étonnamment, plaisent beaucoup plus dans des pays anglophones et n'ont jamais été montées ni publiées en France où il y aurait une façon très différente d'aborder la pièce, sans doute de manière très frontale, très provocatrice, très drag queen. La raison pour laquelle on n'a pas monté Les feluettes en Italie, on me l'a déjà dit, c'est la référence à D'Annunzio. Il y a donc tout un autre bagage culturel qui accompagne la production italienne. On ne peut pas me rendre romantique en Italie avec D'Annunzio, vu son association avec le fascisme.

voix ET IMAGES D'ailleurs, les traductions captent rarement la richesse de cette langue ou la charge émotive d'un langage aux accents familiers pour ne pas dire 
familiaux! Une traduction, toutefois, retient mon attention, justement parce qu'elle capte les nuances de langue: celle en anglais de l'Écosse des Feluettes (The Skelfs).

Michel MARC Bouchard Cette traduction est très réussie. Elle capte bien les différents niveaux de langue, ce qui est impossible d'ailleurs en italien, impossible en espagnol et assez difficile à faire en anglais. C'est d'autant plus marqué parce que je m'amuse beaucoup avec le langage et ce, dans plusieurs de mes pièces; je trouve que le langage est un territoire en soi, comme le personnage. Souvent, il y a une confrontation entre les deux. Dans Des yeux de verre, par exemple, je me suis appliqué à jouer avec quatre niveaux de langue qui évoluent en parallèle, jamais dans l'échange, ce qui s'apparente à une construction mélodique. Comme je le dis souvent aux étudiants que je rencontre, j'écris de la musique avec des rythmes, des tempi, des cadences... La musicalité est une partie fondamentale de mon écriture. Dans Les feluettes, par exemple, il y a cinq niveaux de français que j'ai dû orchestrer pour que tout fonctionne. Car mes personnages se comprennent. Simon ne dit jamais à Vallier qu'il ne comprend rien à ce qu'il dit. Seule Lydie-Anne reprend Simon, mais on comprend bien pourquoi. Il s'agit d'une domination alors qu'entre Vallier et Simon il est plutôt question d'une fascination mutuelle. Mais ces différents niveaux de langue créent de sérieux problèmes de traduction; je sais que Francesca Moccagatta, qui a fait la traduction italienne, s'en arrachait parfois les cheveux.

voIX ET IMAGES Vous avez déjà organisé plusieurs expositions d'art. Pourriez-vous nous parler de l'importance de la peinture et du rôle qu'elle joue dans votre thêâtre en particulier. Je pense notamment au Peintre des madones, mais aussi à toute l'iconographie entourant Saint Sébastien et qui a sans doute nourri votre réflexion pour Les feluettes.

MICHEL MARC BOUCHARD Je n'organise pas d'expositions d'art, mais plutôt des expositions historiques et thématiques. Mon rôle n'est pas le même que celui d'un commissaire d'expositions de beaux-arts. Ces expériences sont distinctes de mon travail théâtral, sauf peut-être pour Ludovica, exposition sur l'histoire de Québec et pour laquelle j'ai écrit dix-neuf saynètes à partir desquelles l'exposition a été montée au Musée de l'Amérique française. Cette exposition a été très bien reçue à Québec et a été reprise par la suite à Bordeaux. Le journal Le Monde a même dit que tous les musées de France devraient s'en inspirer. L'Histoire pour moi est une grande source d'inspiration; c'est peut-être aussi ma façon à moi d'être politique par rapport à la nation. Ne sachant vraiment pas qui je suis, n'étant fait que de paradoxes, j'en cherche l'explication dans mes racines. Alors quand on m'a offert de faire des expositions, j'étais ravi de pouvoir toucher materiellement à l'Histoire par le biais de l'artefact. De plus, ça a été pour moi une grande émotion de pouvoir toucher à l'objet historique, d'être responsable de cet héritage-là, d'avoir la responsabilité de le présenter, de l'interpréter, de lui donner un sens, car contrairement au parti pris d'objectivité d'autres commissaires, ce que je revendique, moi, c'est le parti pris éditorialiste que crée la perspective historique. Je crois qu'avec une exposition, on peut faire reculer les limites de la représentation muséale en dramatisant les sujets.

VoIX ET IMAGES Vous dites que les expositions constituent une œuvre à part, mais on voit quand même des filiations avec votre écriture! Vos expositions sont très théâtrales! 
MICHEL MARC BOUCHARD Oui, peut-être. Les Musées de France ont organisé tout un colloque sur Ludovica pour étudier cette démarche singulière qui intègre une approche théâtrale en muséologie. J'ai aussi présenté sur ce sujet une conférence plénière à l'Association des musées canadiens. Mais c'est sur le tas que j'ai appris comment travailler avec des muséologues. Et je reçois chaque demande d'organiser une exposition comme l'occasion d'élaborer une position éditoriale à partir de mon expérience de dramaturge, certes, mais aussi de metteur en scène. Au musée, le rapport au spectateur est très particulier. Le visiteur n'est pas captif de la salle et peut y passer vingt minutes, une heure ou plus. Et puis, il faut tenir compte des conditions de conservation des objets. Par exemple, rappelons que l'exposition inaugurale de la Grande Bibliothèque, "Tous ces livres sont à toi », a duré quatre mois et demi. Il fallait donc régulièrement tourner les pages des livres pour éviter les dommages causés par la lumière. Le papier, c'est la pire chose à exposer. Le tissu aussi, comme je l'ai découvert avec mon exposition sur les chaussures, Talons et Tentations, au Musée de la Civilisation de Québec.

voix ET IMAGES Soirée bénéfice pour ceux qui ne seront pas là en l'an 2000, toujours inédite, a fait l'objet de plusieurs mises en scène, entre autres au Théâtre du Trillium (Ottawa en 1990) et en 1992 au Théâtre Blanc (Québec). Cette pièce est certainement parmi celles qui ont le plus dérangé. Étrange «conte dramatique», comme vous le dites, cette pièce présente un portrait sauvage et meurtrier de la famille. Pourriezvous nous parler de cette pièce?

MICHEL MARC BOUCHARD D'une part, la pièce est liée à une circonstance personnelle: j'étais alors gravement malade. D'autre part, il y avait des attentes et des craintes qui circulaient à la venue de l'an 2000. Je voulais donc tenter une pièce d'anticipation. Aujourd'hui, je ne sais plus comment parler de cette pièce, parce qu'il y a eu tellement de versions, tellement d'étapes. Et la frustration que j'ai vécue à travers ces étapes-là est maintenant inscrite dans la pièce. Ce qui fait que dans la version actuelle, il y a une très grande violence. Au départ, il y avait un projet très louable d'anticipation, sur un monde où l'homme rencontre la nature et aussi sa propre contre-nature, même dans ses rapports humains. C'était ça, le projet de départ. La pièce a été victime de sa réécriture, de cette expiation de la colère. Je ne sais plus comment parler de cette pièce - quelle est la bonne version, par exemple, quelle est la version définitive? Je me rends compte que toute tentative de réécriture était comme une sorte de tentative de destruction de la première version; si je ne l'ai jamais publiée, c'est que je trouve qu'elle est trop chargée de douleur personnelle. Et je sais que je n'arriverais jamais à corriger les épreuves si un jour il fallait qu'elle paraisse. J'ai accepté, pour la revue, que certains extraits, très près de l'originale, soient publiés. Mais au complet, je ne sais pas...

Voix ET IMAGES Avant même que l'on commence à parler de la privatisation de l'eau et de la surexploitation de nos rivières et de nos cours d'eau, vous en aviez fait un objet de votre réflexion : la guerre de l'eau dans Soirée bénéfice, les déchirements sociopolitiques dans Les porteurs d'eau, l'impact de l'inondation des villages sur la vie des habitants dans Les manuscrits du déluge, le miroir du lac dans Les feluettes, et j'en passe. Depuis au moins Le torrent d'Anne Hébert, l'eau dans son état sauvage est devenu le reflet d'un débordement affectif ou psychique. 
Quelle image émerge de ces multiples traitements de l'eau dans votre dramaturgie?

MICHEL MARC BOUCHARD C'est vrai que ces pièces ont anticipé ce débat-là, mais je dois avouer que ce n'était pas une préoccupation principale ou personnelle. Je peux même dire que j'ai triché! Je m'explique... Parler de la forêt au théâtre, c'était compliqué, parler de l'hiver, c'était encore plus compliqué alors j'ai privilégié l'eau. L'eau est quand même très riche. Il y a une sonorité, il y a des états, il y a des émotions ne serait-ce que par son calme ou par ses rapides, ses déluges. L'eau, c'est aussi les larmes, la naissance, la purification. C'est aussi le pays de rivières et de lacs d'où je viens. De l'eau donc dans son état sauvage : le Lac-Saint-Jean que la Comtesse appelle la Méditerranée dans Les feluettes ou encore la rivière dans Les porteurs d'eau, mais aussi de l'eau "domestiquée». L'eau, c'est aussi un symbole extrêmement catholique : le baptême, la purification. Le bain est un moment porteur de bonheur, comme dans la scène où Maurice amène Teeka «voir la mer» et où on assiste au serment de fidélité, ou dans celle où Vallier et Simon font l'amour dans le bain.

voix ET IMAGES Dans plusieurs de vos pièces, les traumatismes de l'enfance - l'inceste, la violence et l'abandon, entre autres - sont figurés par des variantes d'une même figure: le présentoir de verre dans lequel le corps de la poupée est exposé dans Des yeux de verre, la châsse-maison de Maurice dans L'histoire de l'oie ou encore le "champ de secrets» du triptyque dans Le peintre des madones sont autant de cryptes dans lesquelles est refoulée toute la peine de ceux qui reviennent à ces espaces pour essayer de comprendre.

MICHEL MARC BOUCHARD Je ne sais pas si une blessure traumatique est à la source de cette image récurrente dans mon thêâtre. Je dirais que cette approche-là est plutôt de l'ordre du culturel. Comme je l'ai dit au début de cet entretien, l'Église catholique a été ma première véritable expérience thêâtrale. Or, la messe n'est qu'une série de représentations et de sacralisations d'objets. Tout y est métaphore; c'est d'ailleurs le propre de toute religion. On est loin de l'objet concret. C'est même profondément fétichiste. Il y a cette absence, mais à travers l'objet on a l'impression d'une présence. Dans mon théâtre, il y a souvent l'artefact du souvenir, la robe de la mère, par exemple, dans Les muses orphelines. Dans toutes mes pièces, il y a un moment sacrificiel qui évoque le conflit entre les hommes qui jouent leur destin et les dieux. Cet aspect cérémoniel, référentiel même, est essentiel à toute mon œuvre et c'est par là que celle-ci rejoint, me semble-t-il, l'essence même du thêâtre, qui est rituel et sacrifice du corps de l'acteur. 